\title{
Endogenous GLP1 and GLP1 analogue alter CNS responses to palatable food consumption
}

\author{
Jennifer S ten Kulve', Dick J Veltman², Liselotte van Bloemendaal1, Paul F C Groot3, \\ Henricus G Ruhé4,5, Frederik Barkhof6, Michaela Diamant1, ${ }^{\dagger}$ and Richard G ljzerman' \\ 1Diabetes Center/Department of Internal Medicine, VU University Medical Center, Amsterdam, The Netherlands \\ 2Department of Psychiatry, VU University Medical Center, Amsterdam, The Netherlands \\ 3Department of Radiology, Academic Medical Center, Amsterdam, The Netherlands \\ ${ }^{4}$ Department of Psychiatry, Academic Medical Center, Amsterdam, The Netherlands \\ 5University of Groningen, University Medical Center Groningen, Department of Psychiatry, \\ Groningen, The Netherlands \\ ${ }^{6}$ Department of Radiology \& Nuclear Medicine, VU University Medical Center, Amsterdam, The Netherlands \\ ${ }^{\dagger}$ (Deceased April 2014)
}

Correspondence should be addressed to $\mathrm{J} S$ ten Kulve Email js.tenkulve@vumc.nl

\begin{abstract}
Glucagon-like peptide-1 (GLP1) affects appetite, supposedly mediated via the central nervous system (CNS). In this study, we investigate whether modulation of CNS responses to palatable food consumption may be a mechanism by which GLP1 contributes to the central regulation of feeding. Using functional MRI, we determined the effects of endogenous GLP1 and treatment with the GLP1 analogue liraglutide on CNS activation to chocolate milk receipt. Study 1 included 20 healthy lean individuals and 20 obese patients with type 2 diabetes (T2DM). Scans were performed on two occasions: during infusion of the GLP1 receptor antagonist exendin 9-39 (blocking actions of endogenous GLP1) and during placebo infusion. Study 2 was a randomised, cross-over intervention study carried out in 20 T2DM patients, comparing treatment with liraglutide to insulin, after 10 days and 12 weeks. Compared with lean individuals, T2DM patients showed reduced activation to chocolate milk in right insula $(P=0.04)$. In lean individuals, blockade of endogenous GLP1 effects inhibited activation in bilateral insula $(P \leq 0.03)$. Treatment in T2DM with liraglutide, vs insulin, increased activation to chocolate milk in right insula and caudate nucleus after 10 days $(P \leq 0.03)$; however, these effects ceased to be significant after 12 weeks. Our findings in healthy lean individuals indicate that endogenous GLP1 is involved in the central regulation of feeding by affecting central responsiveness to palatable food consumption. In obese T2DM, treatment with liraglutide may improve the observed deficit in responsiveness to palatable food, which may contribute to the induction of weight loss observed during treatment. However, no long-term effects of liraglutide were observed.
\end{abstract}
Key Words
- fMRI
- GLP1
- obesity
- palatable food
- type 2 diabetes

\section{Introduction}

The central nervous system (CNS) contributes to the maintenance of energy balance and is involved in the regulation of food intake. Altered CNS responses may play an important role in the dysregulation of food intake and consequently in the development of obesity and type 2 diabetes (T2DM) (Volkow \& Wise 2005, Trinko et al. 2007). Several studies on humans revealed that obesity and T2DM are indeed associated with altered CNS activation

Published by Bioscientifica Ltd
Journal of Endocrinology (2016) 229, 1-12 
to food cues (Rothemund et al. 2007, Stoeckel et al. 2008, Stice et al. 2008a,b, van Bloemendaal et al. 2014), as measured with functional magnetic resonance imaging (fMRI). It was shown that obesity is associated with lower responsiveness to palatable food in reward areas of CNS (i.e. caudate nucleus and putamen), which may lead to an increase in food intake, thereby compensating for hypostimulation of the CNS reward system (Stice et al. 2008a,b, Wang et al. 2009). Gaining further insight into the central signalling involved in the evaluation and regulation of food intake is therefore important.

Peripheral signals, hormonal and/or neuronal, convey information about the nutritional status of the body to the CNS, which influences the central regulation of feeding (Schwartz et al. 2000). In the search for therapeutic targets for obesity, several hormones have been explored for their effect on food intake and body weight, however mostly without success (Crawley \& Beinfeld 1983, Costantini et al. 2011, Cummings 2013), except for glucagon-like peptide-1 (GLP1). GLP1 is a gutderived hormone, mainly known for its glucose-lowering effects (Kreymann et al. 1987, Mojsov et al. 1987); however, numerous preclinical and clinical studies have shown that GLP1 has a reducing effect on appetite and feeding (Flint et al. 1998, Rodriquez de et al. 2000, Szayna et al. 2000, Verdich et al. 2001, Chelikani et al. 2005). GLP1 receptor agonists (GLP1RA), used for the treatment of diabetes, have consistently been associated with reduced appetite and weight loss (Verdich et al. 2001, Vilsboll et al. 2012). It is likely that this effect is, at least partly, mediated through the effects of GLP1 on the CNS (Shugrue et al. 1996, Turton et al. 1996, Merchenthaler et al. 1999, Schick et al. 2003, Williams et al. 2009, van Bloemendaal et al. 2014, Secher et al. 2014, Sisley et al. 2014); however, its mechanisms are not fully understood. We previously demonstrated that endogenous GLP1 and administration of GLP1RA affect CNS activation in response to viewing pictures of food items (van Bloemendaal et al. 2014, Ten Kulve et al. 2015a,b), which is related to the predictive value of food consumption and craving for food. However, it is yet unclear whether endogenous GLP1 or treatment with GLP1RA affects CNS responsiveness to the actual palatable food consumption in humans. This is a very important aspect of central food evaluation, as the taste of palatable food can induce an immediate reward that can be a powerful drive for food consumption, therefore leading to an increase in energy intake. Interestingly, GLP1 receptors have not only been found in the CNS (Shugrue et al. 1996, Heppner et al. 2014), but also in the mammalian taste buds
(Shin et al. 2008), and GLP1 signalling was shown to mediate sweet taste perception (Shin et al. 2008). Therefore, we hypothesise that GLP1 and GLP1RA treatment may affect the central processing and reward value of palatable food and that this may be an important mechanism by which GLP1 contributes to the central regulation of feeding in humans.

We have previously shown that acute administration of GLP1RA in obese T2DM patients affects CNS responses to the consumption of palatable food (van Bloemendaal et al. 2015). In the present study on healthy lean individuals and obese patients with T2DM, we determined the effects of endogenous GLP1, using the GLP1 receptor antagonist exendin 9-39, on CNS activation, measured with fMRI, in response to palatable food. We also investigated the effects of treatment with the GLP1RA liraglutide in T2DM patients on CNS activation to palatable food.

\section{Materials and methods}

\section{Subjects}

This study (NCT 01363609) is part of a larger project investigating the effects of GLP1 on the CNS (Ten Kulve et al. 2015a,b). The study was performed in accordance with the Helsinki Declaration and was approved by the Medical Ethics Committee of the VU University Medical Center. All participants provided written informed consent. We included 20 healthy lean individuals and 20 overweight or obese patients with T2DM, matched for gender and age. Participants underwent a screening visit and were eligible if they were 40-65 years of age, right-handed and had a stable body weight $(<5 \%$ reported change during the previous 3 months). Inclusion criteria for healthy lean individuals included a body mass index (BMI) $<25 \mathrm{~kg} / \mathrm{m}^{2}$ and normoglycaemia, defined by fasting plasma glucose $<5.6 \mathrm{mmol} / \mathrm{L}$ and 2 -h glucose $<7.8$ following a $75 \mathrm{~g}$ oral glucose tolerance test. Inclusion criteria for overweight or obese T2DM patients included $\mathrm{BMI}>26 \mathrm{~kg} / \mathrm{m}^{2}$ and HbA1c levels between 42 and $69 \mathrm{mmol} / \mathrm{mol}$ (6.0-8.5\%). For the current treatment of diabetes, only the oral glucose-lowering agents metformin \pm sulphonylurea derivatives were allowed. Exclusion criteria for all subjects were a history of neurological, cardiovascular, renal or liver disease, malignancies, the use of any centrally acting agent or oral glucocorticoids, substance abuse and psychiatric disorders (including eating disorders and depression, assessed with the Eating Disorder Inventory II and the Center for Epidemiologic Studies Depression scale

Published by Bioscientifica Ltd. 
respectively (Garner \& Olmsted 1986, Schroevers et al. 2000)) and the inability to undergo MRI scanning.

All T2DM patients were treated for diabetes with metformin and 12 patients were also treated with sulphonylurea. Sulphonylurea drugs were temporarily discontinued 4 weeks before the start of the experiments, but treatment with metformin was continued. Ten patients used antihypertensive medication and 15 patients used cholesterol-lowering agents.

\section{General experimental protocol}

Study 1: endogenous GLP1 study Both healthy lean individuals and T2DM patients participated in this randomised, placebo-controlled, acute intervention study. The experimental protocol of this study has been described in detail previously (Ten Kulve et al. 2015a). Briefly, two visits were scheduled and participants arrived in the morning at the research unit after an overnight fast. A catheter was inserted into a cubital vein for infusion (in random order) of either placebo ( $0.9 \%$ sodium chloride solution) or the GLP1 receptor antagonist exendin 9-39 (Bachem; Clinalfa products, Bubendorf, Switzerland) at a rate of $600 \mathrm{pmol} / \mathrm{kg} / \mathrm{min}$ using a MRI-compatible infusion pump (MRIdium 3850 MRI IV pump, Iradimed, Winter Park, USA). Each infusion (placebo or exendin 9-39) started $2 \mathrm{~h}$ before the beginning of the standardised meal intake and was continued during the entire MR scanning period. MR imaging was performed $45 \mathrm{~min}$ after the intake of a standardised liquid meal $(450 \mathrm{kcal}$, carbohydrate $56.1 \mathrm{~g}$, fat $17.4 \mathrm{~g}$ and protein $18.0 \mathrm{~g} ; 300 \mathrm{~mL}$ Nutridrink yoghurt style, raspberry flavour; Nutricia, Zoetermeer, The Netherlands) in order to induce endogenous GLP1 secretion. The participants were blinded for the type of infusion. Blood was drawn at the start of the test visit and every $30 \mathrm{~min}$ from the start of the standardised meal intake until 60 min to measure glucose, GLP1, insulin and glucagon levels.

\section{Study 2: Treatment effects of liraglutide vs} insulin glargine After study 1, we included the T2DM patients in a randomised, cross-over intervention study. The experimental protocol of this study has been described in detail previously (Ten Kulve et al. 2015b). Briefly, the study consisted of two treatment periods of 12 weeks each with a 12-week washout period in between. During one period, patients were treated with liraglutide, with a dose-escalation period, starting at $0.6 \mathrm{mg}$ once daily (q.d.), with weekly increments of $0.6 \mathrm{mg}$, if well tolerated, reaching a final dose of $1.8 \mathrm{mg}$ q.d. by the end of the second week. During the other treatment period, patients were treated with an active comparator, i.e. insulin glargine, started at an initial dose of $10 \mathrm{IU}$ q.d. with dose-escalation period based on their fasting self-monitored blood glucose levels according to a predetermined treat-to-target algorithm (Bunck et al. 2011). The order of treatment was determined by block randomisation, with a block size of four.

Two series of three visits with MRI sessions were scheduled. For each treatment, one at the start (baseline), one after 10 days (short term) of each treatment and one after 12 weeks (long term). All visits were similar to those described in study 1, however without exendin 9-39 infusion. At each visit, anthropometric measurements were performed, body composition was measured using bioelectrical impedance analysis and a blood sample was taken for HbA1c and lipid measurements. Nausea was measured during each test visit at five fixed time points using a ten-point Likert scale.

\section{fMRI paradigm}

We previously investigated and described the results from these studies, presenting the effects of endogenous GLP1 and treatment with GLP1RA liraglutide on the CNS activation to visual food cues, i.e. viewing food pictures (Ten Kulve et al. 2015a,b). The current fMRI paradigm was developed to investigate the CNS activation to actual consumption of palatable food and has been described previously (van Bloemendaal et al. 2015). In short, chocolate milk (Chocomel, FrieslandCampina, per $100 \mathrm{~mL}$ : $86 \mathrm{kcal}, 2.7 \mathrm{~g}$ fat, $11.8 \mathrm{~g}$ sugar) was used as a palatable food stimulus. As a neutral stimulus, a tasteless solution was used, designed to mimic the natural taste of saliva (consisting of $2.5 \mathrm{mM} \mathrm{NaHCO} 3$ and $25 \mathrm{mM}$ $\mathrm{KCl}$ (Stice et al. 2008a)). This solution should provide a superior neutral condition compared with water, which has a taste that activates the gustatory cortex (Zald \& Pardo 2000, O'Doherty et al. 2001). Participants received $0.4 \mathrm{~mL}$ of the chocolate milk or tasteless solution per 'trial'. In each trial, participants were presented a picture of a orange triangle (coupled to chocolate milk) or a blue star (coupled to tasteless solution), which was followed by the receipt of the coupled solution. Participants were instructed to keep the solution within their mouth for $6 \mathrm{~s}$ until the sign 'swallow' was presented. The described trials were the paired trials. However, on $40 \%$ of the trials, the solution was not delivered (unpaired trails), in order to maintain an unconditioned response to the receipt of

Published by Bioscientifica Ltd 
the solution. At the beginning of each trial, subjects were unaware whether the trial was paired or unpaired, and the order of trials was randomised. Figure 1 depicts the different trials and events.

The task was programmed and performed using E-prime 1.2 (Psychology Software Tools, Inc., Pittsburg, PA, USA). The taste solutions were delivered with two programmable infusion pumps (B. Braun, Infusomat $\mathrm{P}$, Melsungen, Germany). The infusion pumps were connected with Vygon tubing, which was attached to the MRI head coil and inserted into the participant's mouth.

\section{MRI acquisition and analyses}

MRI acquisition methods have been described previously (van Bloemendaal et al. 2014). In short, scans were acquired on a 3.0 Tesla GE Signa HDxt scanner (General Electric). Structural MRI was obtained using a T1-weighted sequence. FMRI data were acquired using an echo planar imaging T2* blood oxygen level-dependent pulse sequence with whole-brain coverage. Functional images were pre-processed and analysed with SPM8 software (Wellcome Trust Centre for Neuroimaging, London, UK).

Functional scans were analysed in the context of general linear model. The receipt of chocolate milk and tasteless solution was considered as an event of interest and was modelled at the first (single-subject) level. Next, two contrast images were computed for each subject and each scan, i.e. 'chocolate milk vs baseline', to determine in which areas activation was greater during chocolate milk receipt compared with during baseline, and 'chocolate milk vs tasteless solution', to narrow down the effects of chocolate milk receipt, as this contrast determined in which areas activation was greater during chocolate milk receipt compared with tasteless solution receipt, thus identifying only the added effect of the receipt of palatable solution (i.e. chocolate milk) and eliminating the effect of the receipt of a solution in general.

These first-level contrast images were entered into second-level two-way ANOVA. The model included the factor group (healthy lean, T2DM) and infusion (placebo, exendin 9-39) for study 1 and liraglutide and insulin glargine treatment and time point (baseline, 10 days and 12 weeks of treatment) for study 2.

\section{Blood sampling and assays}

The measurement of blood glucose (study 1 and 2) was performed immediately after collection using the glucose dehydrogenase method (GlucoseAnalyzer, HemoCue, Ängelholm, Sweden). For other analyses, blood samples were cold centrifuged at $-4^{\circ} \mathrm{C}$ directly after collection and plasma was stored at $-80^{\circ} \mathrm{C}$. Glucagon levels were determined using an immunoassay, as described previously (Lilly Research Laboratories, Indianapolis, IN, USA) (Sloan et al. 2012). Total GLP1 was analysed using a C-terminally directed radioimmunoassay for amidated GLP1 (antibody 89390 (Orskov et al. 1994)). Insulin levels were measured using an immunometric assay (Advia, Centaur, Siemens Medical Solutions Diagnostics,

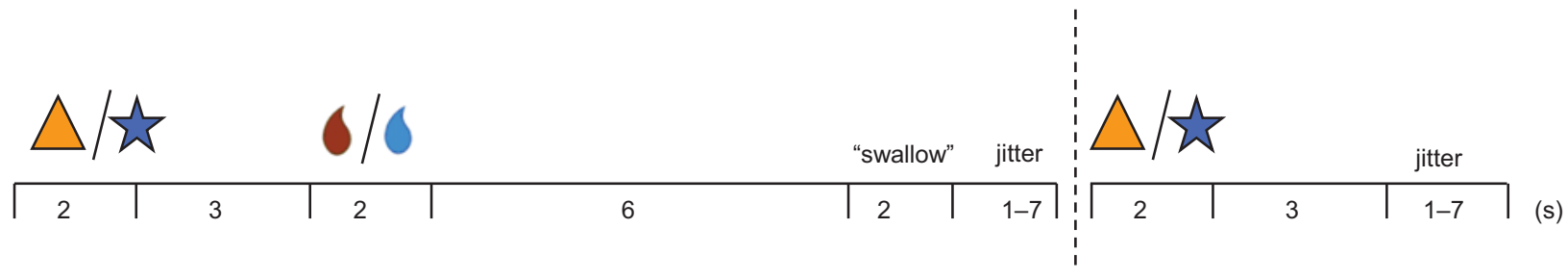

\section{Figure 1}

fMRI paradigm of chocolate milk and tasteless solution receipt. The fMRI paradigm consisted of two types of trials: paired and unpaired trials, which were randomised in order and type. Paired trials: Subjects were presented a picture of an orange triangle or a blue star for 2 s. The orange triangle was coupled to chocolate milk receipt and the blue star to tasteless solution receipt. After the presentation of the picture, the subjects waited for $3 \mathrm{~s}$, while watching a fixation cross, until receiving the coupled solution (for $2 \mathrm{~s}$ ). The subjects were instructed to keep the solution within the mouth for $6 \mathrm{~s}$ and to refrain from swallowing until the sign 'swallow' was presented afterwards. Between the trials, a jitter of 1-7 s was used. In total, 40 paired trials were presented and half of them included the orange triangle and chocolate milk receipt. Unpaired trials: These trials were similar to the beginning of the paired trial, however without receiving the coupled solution. Between the trials, a jitter of 1-7 s was used. At the beginning of the trials, subjects were unaware whether it consisted a paired or unpaired trials. In total, 32 paired trials were presented and half of them included the orange triangle. A full colour version of this figure is available at http://dx.doi.org/10.1530/JOE-15-0461. 
Malvern, PA, USA). Total insulin, glucagon and GLP1 levels were only determined in study 1 .

\section{Statistical analyses}

Imaging data were analysed with SPM8. In study 1, between-group differences as well as effects within group of infusion were assessed. In study 2, differences at the three time points between treatments were assessed. A priori regions of interest were determined based on the previous studies (i.e. insula, striatum, amygdala and OFC) (Rothemund et al. 2007, Stoeckel et al. 2008, Tang et al. 2012, van Bloemendaal et al. 2014). CNS activations were reported as significant when these survived familywise error correction for multiple comparisons at the voxel level using small volume correction, as described previously (van Bloemendaal et al. 2014).

Clinical group data and treatment effects were analysed using Statistical Package for the Social Sciences version 20 (SPSS20). Data are expressed as mean \pm s.E.M. (unless otherwise stated). In study 1, between-group differences were analysed with independent Student's $t$-tests or, in case of more than one time point during one visit, with repeated measures ANOVA using time (minutes) as within-subject factor and group as between-subject factor. In study 2 , to analyse the longitudinal difference between treatments, a generalised estimating equation approach was used. To investigate the effect of order of treatment on the changes in weight and HbA1c, an interaction term was added to the model. Associations among differences in CNS activation between treatments and clinical data were calculated with Pearson's regression coefficient. Results were considered statistically significant when $P<0.05$.

\section{Results}

\section{Clinical characteristics, blood glucose and plasma hormone levels}

Table 1 summarises the clinical characteristics of both groups from study 1, as described previously (Ten Kulve et al. 2015a). We included 20 obese patients with T2DM and 20 age- and gender-matched healthy lean individuals. All participants completed all visits. By design, the groups differed on weight, BMI, waist circumference, fasting plasma glucose and HbA1c $(P<0.001)$. Furthermore, systolic blood pressure was significantly higher in T2DM patients.

The glucose and hormone responses during the test visits of study 1 are shown in Supplementary Fig. 1 (see section on supplementary data given at the end of this article) (as described previously (Ten Kulve et al. 2015a)). As expected, T2DM patients had significantly higher glucose and glucagon levels compared with healthy lean individuals. Exendin 9-39 infusion, compared with placebo, had no effects on glucose levels in healthy lean individuals, but increased glucose levels in T2DM patients and increased glucagon levels in both groups. GLP1 levels did not differ significantly between healthy lean individuals and T2DM patients during placebo infusion but were significantly higher during the exendin 9-39 infusion compared with placebo.

In study 2, the effects of treatment with liraglutide and insulin glargine on clinical characteristics in T2DM patients are presented in Table 2 , as described previously (Ten Kulve et al. 2015b). All participants completed all the visits. Treatment with liraglutide resulted in significant weight loss after 12 weeks compared with insulin

Table 1 Clinical characteristics of healthy lean individuals and T2DM patients.

\begin{tabular}{|c|c|c|c|}
\hline & $\begin{array}{l}\text { Healthy controls } \\
\qquad(n=20)\end{array}$ & $\begin{array}{c}\text { Obese patients } \\
\text { with T2DM }(n=20)\end{array}$ & P-value \\
\hline Age (years) & $56.3 \pm 1.4$ & $59.5 \pm 0.9$ & 0.06 \\
\hline Gender, male/female $(n)$ & $10 / 10$ & $11 / 9$ & 0.8 \\
\hline Weight $(\mathrm{kg})$ & $69.9 \pm 2.5$ & $95.4 \pm 3.4$ & $<0.001$ \\
\hline Body mass index $\left(\mathrm{kg} / \mathrm{m}^{2}\right)$ & $22.5 \pm 0.4$ & $32.0 \pm 1.1$ & $<0.001$ \\
\hline Waist circumference $(\mathrm{cm})$ & $81.4 \pm 1.8$ & $108.9 \pm 2.5$ & $<0.001$ \\
\hline Systolic blood pressure $(\mathrm{mmHg})$ & $113 \pm 3.5$ & $128 \pm 2.0$ & 0.001 \\
\hline Diastolic blood pressure $(\mathrm{mmHg})$ & $72.9 \pm 2.5$ & $78 \pm 1.8$ & 0.1 \\
\hline $\mathrm{HbA} 1 \mathrm{c}(\mathrm{mmol} / \mathrm{mol})$ & $37 \pm 0.4$ & $56 \pm 2.2$ & $<0.001$ \\
\hline Fasting plasma glucose (mmol/L) & $5.2 \pm 0.1$ & $8.4 \pm 0.3$ & $<0.001$ \\
\hline Diabetes duration (years) & - & $7.8 \pm 1.1$ & - \\
\hline Blood pressure-lowering medications $(n)$ & 0 & 10 & $<0.001$ \\
\hline Cholesterol-lowering medications $(n)$ & 0 & 15 & $<0.001$ \\
\hline
\end{tabular}

Data are means \pm S.E.M.

T2DM, type 2 diabetes.

http://joe.endocrinology-journals.org DOI: $10.1530 / \mathrm{JOE}-15-0461$
(C) 2016 Society for Endocrinology Printed in Great Britain 
Table 2 Clinical characteristics before and during treatment in obese T2DM patients.

\begin{tabular}{l} 
Patient characteristics $(n=20)$ \\
\hline Weight $(\mathrm{kg})$ \\
BMI $\left(\mathrm{kg} / \mathrm{m}^{2}\right)$ \\
Waist circumference $(\mathrm{cm})$ \\
Systolic blood pressure $(\mathrm{mmHg})$ \\
Diastolic blood pressure $(\mathrm{mmHg})$ \\
Heart rate $($ beats/min) \\
HbA1c (mmol/L) \\
Fasting glucose $(\mathrm{mmol} / \mathrm{L})$ \\
Total cholesterol $(\mathrm{mmol} / \mathrm{L})$ \\
Triglycerides $(\mathrm{mmol} / \mathrm{L})$ \\
Nausea score
\end{tabular}

\begin{tabular}{ccc}
\hline \multicolumn{2}{c}{ Baseline } \\
\cline { 1 - 2 } Insulin & & Liraglutide \\
\cline { 1 - 1 } $94.6 \pm 3.5$ & & $95.0 \pm 3.4$ \\
$31.7 \pm 1.1$ & & $31.9 \pm 1.0$ \\
$108 \pm 2.5$ & & $108 \pm 2.2$ \\
$126 \pm 1.8$ & & $127 \pm 1.9$ \\
$78 \pm 1.9$ & & $79 \pm 3.2$ \\
$67 \pm 1.7$ & & $68 \pm 2.4$ \\
$54 \pm 1.8$ & & $55 \pm 2.0$ \\
$8.1 \pm 0.4$ & & $8.3 \pm 0.4$ \\
$4.6 \pm 0.3$ & & $4.2 \pm 0.2$ \\
$1.8 \pm 0.3$ & & $1.6 \pm 0.2$ \\
$0.37 \pm 0.16$ & & $0.76 \pm 0.37$
\end{tabular}

\begin{tabular}{ccc}
\hline \multicolumn{2}{c}{10 days } \\
\cline { 1 - 2 } Insulin & & Liraglutide \\
\cline { 1 - 1 } $94.4 \pm 3.5$ & & $93.9 \pm 3.4$ \\
$31.6 \pm 1.1$ & & $31.5 \pm 1.0$ \\
$108 \pm 2.4$ & & $108 \pm 2.2$ \\
$125 \pm 2.4$ & & $125 \pm 1.9$ \\
$77 \pm 1.6$ & & $77 \pm 1.9$ \\
$67 \pm 2.0$ & & $72 \pm 1.8^{*}$ \\
$54 \pm 2.0$ & & $54 \pm 2.2$ \\
$6.8 \pm 0.3$ & & $6.0 \pm 0.3^{\dagger}$ \\
$4.3 \pm 0.3$ & & $4.0 \pm 0.3$ \\
$1.4 \pm 0.1$ & & $1.4 \pm 0.1$ \\
$0.35 \pm 0.24$ & & $0.51 \pm 0.20$ \\
\hline
\end{tabular}

\begin{tabular}{ccc}
\hline \multicolumn{2}{c}{12 weeks } \\
\cline { 1 - 2 } Insulin & Liraglutide \\
\cline { 1 - 1 } \cline { 1 - 1 } $95.4 \pm 3.6$ & & $91.7 \pm 3.5^{\dagger}$ \\
$31.9 \pm 1.0$ & & $30.7 \pm 1.0^{+}$ \\
$108 \pm 2.4$ & & $105 \pm 2.3^{+}$ \\
$125 \pm 2.5$ & & $123 \pm 1.9$ \\
$76 \pm 1.8$ & & $76 \pm 1.5$ \\
$67 \pm 2.4$ & & $71 \pm 1.7$ \\
$51 \pm 1.7$ & & $47 \pm 1.6^{+}$ \\
$5.2 \pm 0.2$ & & $6.2 \pm 0.3^{+}$ \\
$4.3 \pm 0.3$ & & $4.0 \pm 0.2$ \\
$1.4 \pm 0.2$ & & $1.4 \pm 0.2$ \\
$0.36 \pm 0.17$ & $0.33 \pm 0.16$
\end{tabular}

Data are means \pm S.E.M.; $* P<0.05 ;{ }^{\dagger} P<0.001$ for differences between liraglutide and insulin glargine treatment change from baseline. T2DM, type 2 diabetes.

glargine ( $\Delta-3.3 \mathrm{~kg}$ vs $\Delta+0.8 \mathrm{~kg}$, respectively, $P<0.001)$. HbA1c levels decreased during both treatments, however, significantly more with liraglutide compared with placebo $(\Delta-8 \mathrm{mmol} / \mathrm{L}(-0.7 \%)$ vs $-3 \mathrm{mmol} / \mathrm{L}(-0.2 \%)$, respectively, $P<0.001)$. There was no significant interaction between the order of treatment and the effect of treatment on both body weight and HbA1c levels $(P=0.1$ and $P=0.3$ respectively). Supplementary Fig. 2 shows that both liraglutide and insulin glargine lowered glucose levels compared with baseline $(P<0.001)$; however, this effect was larger with liraglutide compared with insulin glargine. Nausea scores during the test visits did not differ between treatments after 10 days or 12 weeks ( $P=0.5$ and $P=0.4$ respectively).

\section{Decreased CNS activation in response to chocolate milk receipt in obese T2DM patients vs healthy lean individuals}

Supplementary Table 1 (see section on supplementary data given at the end of this article) shows the results of the effects of group on CNS activation in response to chocolate milk receipt. Obese T2DM patients, compared with healthy lean individuals, showed less CNS activation in right insula $(P=0.04)$ and left OFC $(P=0.003)$ in response to chocolate milk (Fig. 2). In the analyses using the contrast chocolate milk receipt vs tasteless solution receipt, similar results were observed in right insula $(P=0.04)$.
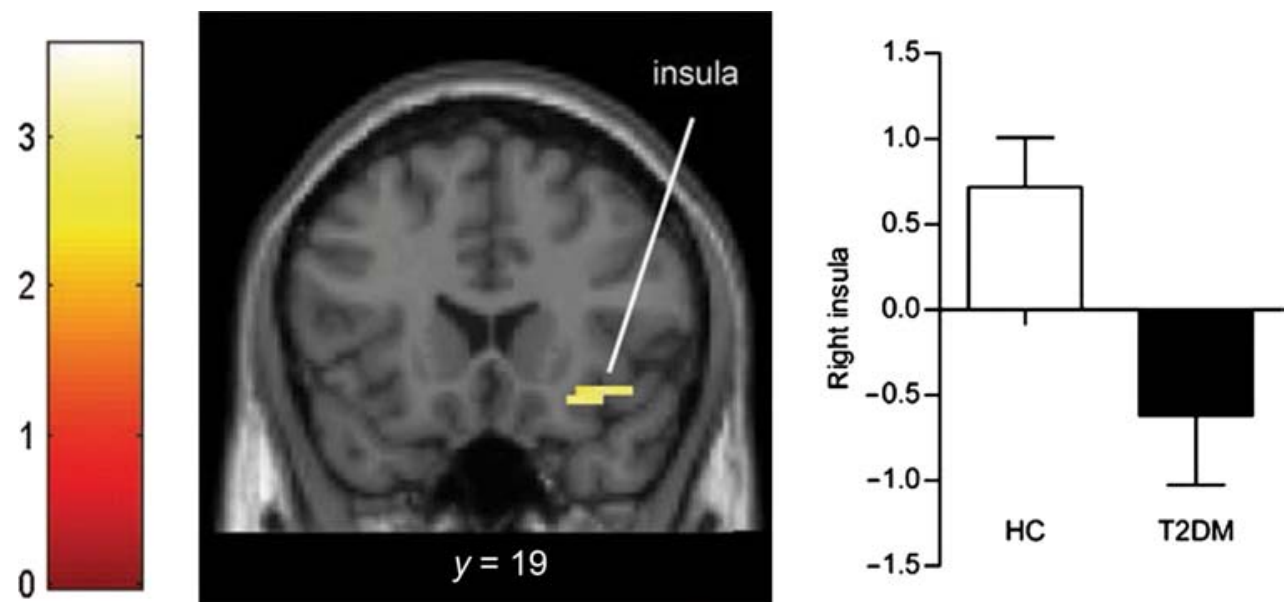

Figure 2

Group differences on CNS activation to chocolate milk receipt. Coronal slice showing differences in group averages of activation in brain regions where T2DM patients $(n=20)$ vs healthy lean individuals $(n=20)$ had reduced activation in response to chocolate milk receipt. The colour scale reflects the $T$ value of the functional activity. Results are presented at the threshold of $P<0.05$, FWE corrected (correction for multiple comparisons on the voxel level) on cluster extent. In the graphs, BOLD signal intensity (effect size) is plotted (arbitrary unites), mean \pm S.E.M. BOLD, blood oxygen level dependent; FWE, family-wise error; HC, healthy lean individuals; T2DM, type 2 diabetes patients. A full colour version of this figure is available at http://dx.doi.org/10.1530/JOE-15-0461. 


\section{Study 1: Endogenous GLP1 affects CNS responses to chocolate milk receipt in healthy lean individuals}

In healthy lean individuals, blockade of endogenous GLP1 effects with the GLP1 receptor antagonist exendin 9-39 resulted in a blunted CNS activation in response to the receipt of chocolate milk in bilateral insula (right insula, $P=0.03$; left insula, $P=0.02$ ) (Fig. 3). In T2DM patients, we did not detect an effect of blockade of the GLP1 receptor. In the analyses with the use of the contrast chocolate milk vs tasteless solution, comparable effects of the GLP1 antagonist were found in the healthy lean individuals (right insula, $P=0.01$; left insula, $P=0.03$ ), and again no effects were observed in T2DM patients (Supplemental Table 1).

\section{Study 2: Treatment with liraglutide vs insulin glargine increases CNS activation to chocolate milk receipt after short-term treatment}

We compared the effects of liraglutide and insulin glargine at baseline, after 10 days and after 12 weeks of treatment in a cross-over study. As expected, no differences were observed between treatments at baseline in response to chocolate milk receipt. After 10 days of treatment with liraglutide, compared with insulin glargine, CNS activation to chocolate milk compared with baseline was increased in right caudate nucleus $(P=0.013)$ and right insula $(P=0.032)$ (Fig. 4). After 12 weeks, we did not observe the differences between treatments in our regions of interest. However, insulin glargine, in comparison to liraglutide, increased the activation in response to chocolate milk in right amygdala region $(P=0.001)$ (Supplementary Table 1).
We also compared the effects of liraglutide and insulin glargine using the contrast chocolate milk vs tasteless solution receipt. Again as expected, we did not observe the differences between treatments at baseline. However, after 10 days of treatment, there were no differences between treatments. After 12 weeks, results with this contrast were similar to those obtained with the contrast chocolate milk receipt vs baseline, with increased activation with insulin glargine in response to chocolate milk in right amygdala region $(P=0.001)$.

\section{Correlation between differences of treatment in CNS activation and weight changes}

The difference between treatments (after 10 days) in CNS activation to the receipt of chocolate milk in the caudate nucleus and the right insula was positively correlated with the difference in weight change between treatments after 12 weeks; however, this was only statistically significant for the right insula (right caudate nucleus $r=0.4, P=0.1$; right insula $r=0.5, P=0.03$ ) (Fig. 5).

\section{Adverse events}

In study 1 , four individuals reported mild abdominal discomfort after meal intake (one during exendin 9-39, one during placebo and two during both visits) (Ten Kulve et al. 2015a). During exendin 9-39 infusion, one individual vomited shortly after the intake of meal and another individual experienced dizziness after the first fMRI session.
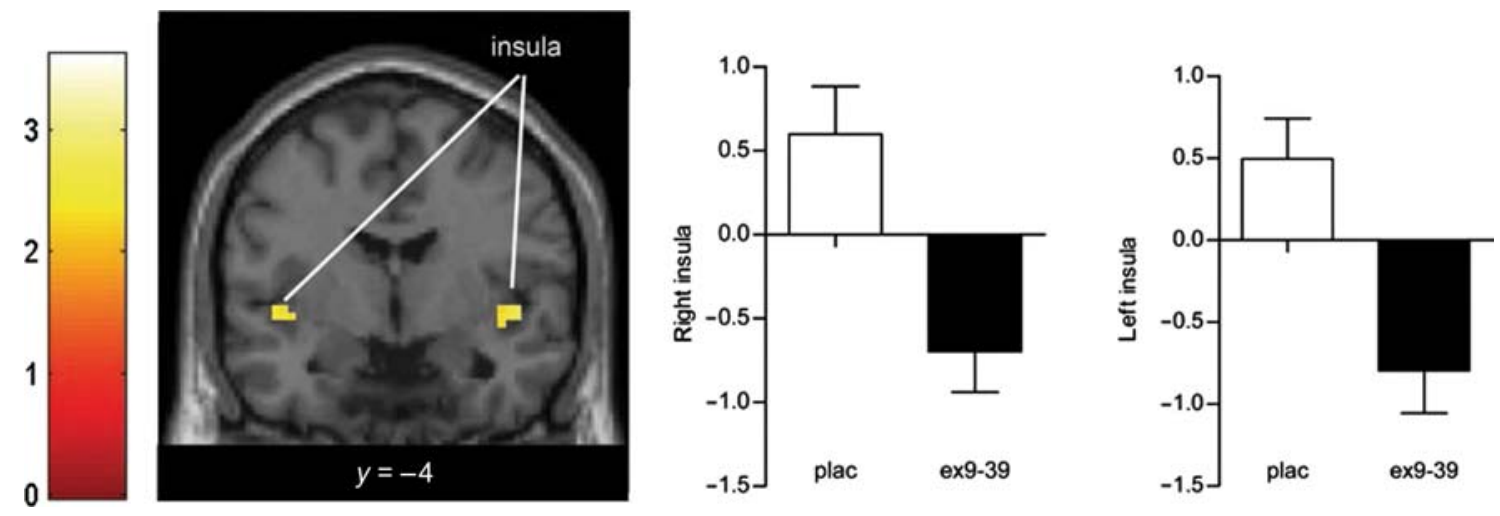

\section{Figure 3}

Effects of endogenous GLP1 on CNS activation to chocolate milk receipt. Coronal slice showing the areas where $(n=20)$ blockade of endogenous GLP1 effects reduced the activation in response to chocolate milk receipt in healthy lean individuals. The colour scale reflects the $T$ value of the functional activity. Results are presented at the threshold of $P<0.05$, FWE corrected (correction for multiple comparisons on the voxel level) on cluster extent. In the graphs, BOLD signal intensity (effect size) is plotted (arbitrary unites), mean \pm s.E.M. BOLD, blood oxygen level dependent; ex9-39, exendin 9-39; FWE, family-wise error; GLP1, glucagon-like peptide-1; plac, placebo. A full colour version of this figure is available at http://dx.doi.org/10.1530/JOE-15-0461. 


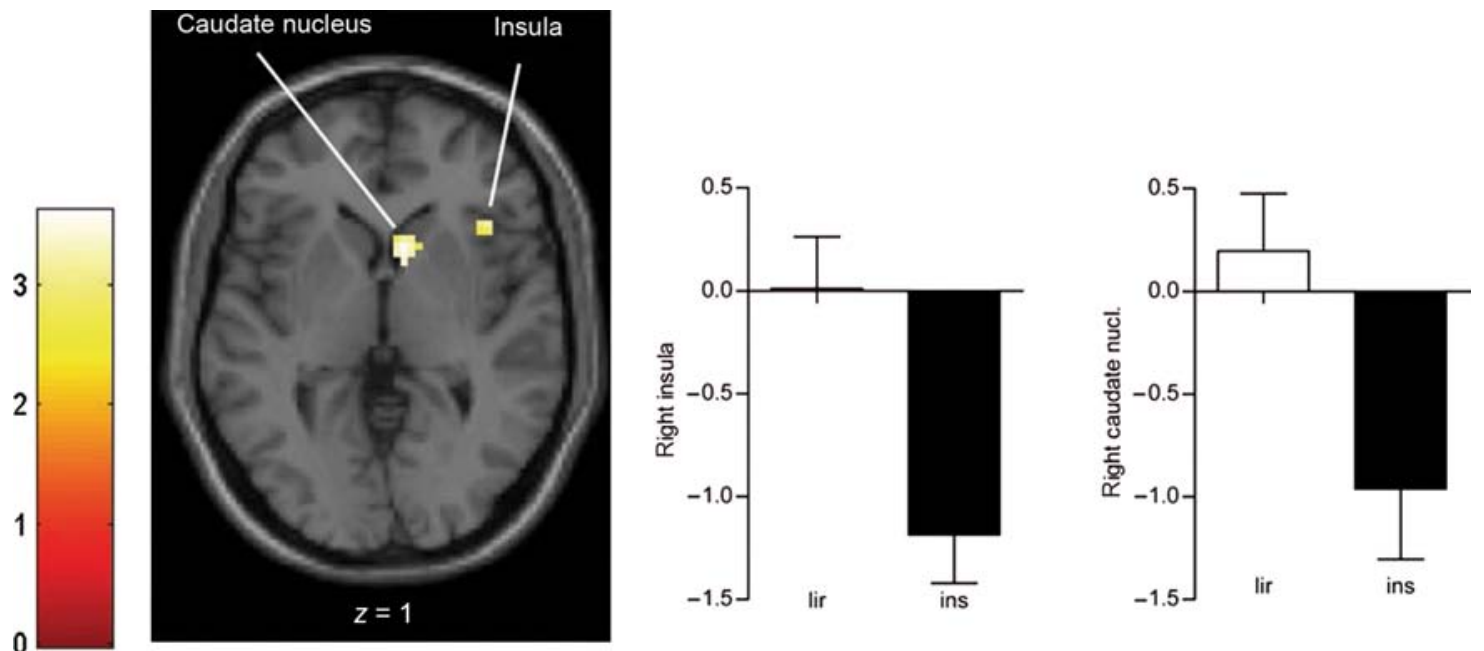

\section{Figure 4}

Effects of GLP1RA treatment in obese T2DM patients on CNS activation to chocolate milk receipt. Axial slice showing areas where after 10 days of treatment with liraglutide vs insulin, increased responses were observed in response to chocolate milk receipt in obese T2DM patients. The colour scale reflects the $T$ value of the functional activity. Results are presented at the threshold of $P<0.05$, FWE corrected (correction for multiple comparisons on the voxel level) on cluster extent. In the graphs, BOLD signal intensity (effect size) is plotted (arbitrary unites), mean \pm s.E.M. BOLD, blood oxygen level dependent; FWE, family-wise error; GLP1, glucagon-like peptide-1; T2DM, type 2 diabetes patients. A full colour version of this figure is available at http://dx.doi.org/10.1530/JOE-15-0461.

In study 2, during treatment with insulin glargine, three patients reported one or more mild hypoglycaemic episodes (Ten Kulve et al. 2015b). During treatment with liraglutide, one patient reported a mild hypoglycaemic episode. During the first days of treatment or after dose escalation with liraglutide, ten patients reported gastro-intestinal side effects (mild nausea, $n=7$; moderate nausea, $n=1$; diarrhoea, $n=2$ ). At the beginning of the test visit after 10 days of treatment with liraglutide, two patients reported a mild feeling of nausea. When we excluded these patients from the analyses at that time point, the results of the effect of liraglutide on CNS activation remained similar.

\section{Discussion}

Previously, we demonstrated the effects of endogenous GLP1 and treatment with GLP1RA on CNS activation in response to viewing food pictures. This study was performed to investigate the effects of endogenous GLP1 and treatment with GLP1RA on CNS activation in response to palatable food consumption, as the taste of food can induce reward-related CNS responses, which can be a powerful drive for food consumption. We found that healthy lean individuals have greater activation, compared with obese T2DM patients, in the insula in response to receipt of chocolate milk. In healthy lean individuals, blockade of endogenous GLP1 effects precluded these activations in the insula. In obese T2DM patients, 10 days of treatment with the GLP1RA liraglutide, compared with insulin, improved the reduced activation in the insula and caudate nucleus in response to chocolate milk receipt of which the insula activation predicted decreases in body weight after 12 weeks. This suggests that the GLP1RA liraglutide may improve the observed deficit in responsiveness in T2DM compared with lean individuals. However, this treatment effect on the CNS ceased to be significant after long-term treatment (12 weeks). Together, these findings indicate that endogenous GLP1 modulates central responses to palatable food consumption and that short-term treatment with GLP1RA may normalise CNS responses to palatable food consumption in obese T2DM individuals.

It has been suggested that altered CNS responses may contribute to the development of obesity (Volkow \& Wise 2005, Trinko et al. 2007). These altered CNS responses in obesity to food stimuli often parallel observations in individuals with drug addiction in response to addictionrelated stimuli. Obese individuals showed increased responsiveness to viewing pictures of food, which could be regarded as the addictive substance, in areas involved in reward circuits (such as the insula, putamen, caudate nucleus and amygdala) (Rothemund et al. 2007, Stoeckel et al. 2008, van Bloemendaal et al. 2014), which may resemble craving effects. They also showed a reduced responsiveness to actual receipt of palatable food in the

Published by Bioscientifica Ltd. 
A
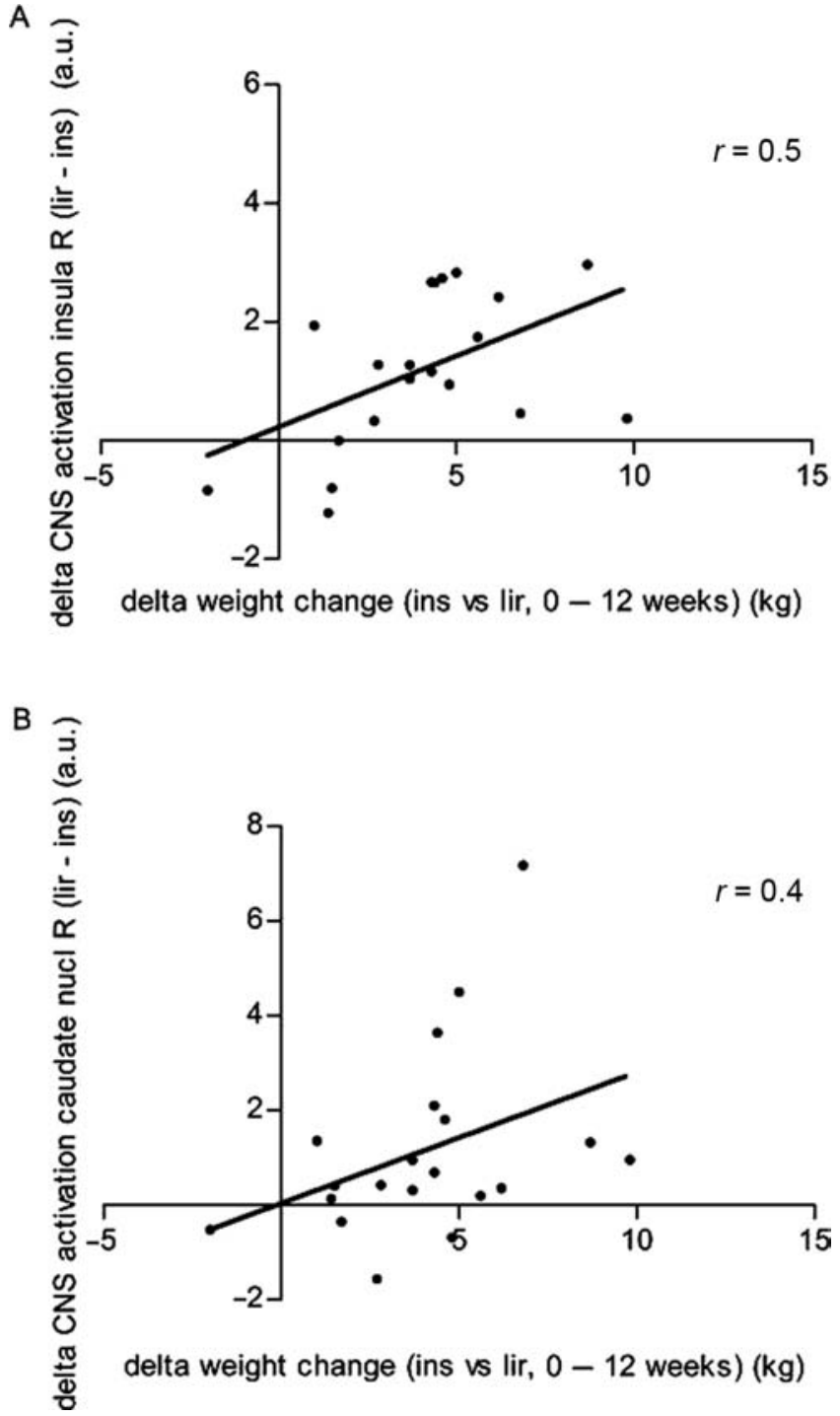

Figure 5

Correlation between difference in CNS activation in (A) the right insula and (B) the right caudate nucleus after 10 days and difference in weight changes over 12 weeks between treatments. Correlation analyses were performed between the differences in CNS activation in response to chocolate milk receipt after 10 days of treatment with liraglutide vs insulin and the differences between treatments in weight change from baseline to 12 weeks. A positive correlation was found in both areas where a difference was observed of liraglutide vs insulin in response to chocolate milk response after 10 days of treatment (insula, $r=0.5$; caudate nucleus, $r=0.4$ ); however, this was only significant for the activation in the insula (insula, $P=0.03$; caudate nucleus, $P=0.1$ ).

caudatenucleusand putamen, probablyreflecting (acquired or premorbid) hyposensitivity (Stice et al. 2008a,b). In line with these findings, we observed a reduced responsiveness to the receipt of palatable food in our group of obese T2DM patients. This deficit in responsiveness of the reward circuitry to palatable food intake may lead to an increase in food intake, as a means to compensate for under-stimulation of the CNS reward system, in an effort to achieve a sufficient degree of satisfaction (Blum et al. 1996, Wang et al. 2004). Interestingly, we found that short-term treatment with the GLP1RA liraglutide, compared with insulin, increased the responsiveness to palatable food consumption in the insula and caudate nucleus in T2DM, suggesting improved stimulation in the reward circuitry, which may prevent the compensatory increase in food intake.

The insula is important in the reward evaluation of food consumption, therefore the regulation of food intake. The insula is known to receive gustatory and visceral afferents, therefore regarded as the primary gustatory cortex, and to be involved in taste memory (Levy et al. 1999). It is involved in the processing and evaluation of rewarding aspects of food cues and in craving for food (Small et al. 2001, Pelchat et al. 2004). Moreover, the insula is connected to other reward-related areas (Frank et al. 2013). Also the caudate nucleus is known to be involved in the process of reward evaluation (Hare et al. 2008). Our findings of endogenous GLP1 effects and treatment with liraglutide in these areas in response to chocolate milk consumption indicate a role for GLP1 in the central regulation of feeding behaviour.

In contrast to the healthy lean individuals, an effect of endogenous GLP1 on chocolate milk receipt was not observed in T2DM patients. In these patients, CNS activation to the receipt of chocolate milk during placebo infusion was lower compared with the healthy lean individuals. It is possible that this lower activation in T2DM patients might have hampered the likelihood to detect a further decrease caused by blockade of endogenous GLP1 effects. The absence of measurable effect of endogenous GLP1 on CNS activation in T2DM patients cannot be explained by lower GLP1 levels, as GLP1 levels did not differ between groups.

Although we found an effect of treatment with liraglutide after short-term treatment, we did not observe an effect after 12 weeks. Because the effects on the CNS precede the weight reduction, it therefore may be suggested that effects of liraglutide on the CNS contribute to the induction of weight loss, as seen during the initial period of treatment; however, it is unclear whether the effects on the CNS contribute to the maintenance of weight loss. Results from clinical studies show that the weight loss observed during treatment with liraglutide $(1.8 \mathrm{mg})$ is most pronounced in the first 10 weeks, but is only maintained during long-term treatment (Buse et al. 2009, Russell-Jones et al. 2009). As we could not detect an enhancing effect of liraglutide after long-term treatment

Published by Bioscientifica Ltd. 
on the CNS, it is to suggest that liraglutide maintains weight loss via other mechanisms than those involving the CNS or that the effects on the CNS may be smaller after long-term treatment. This may explain why the weight loss does not proceed after long-term treatment.

For the effects of T2DM and endogenous GLP1, the results were similar using the contrast chocolate milk receipt vs baseline and using the contrast chocolate milk vs tasteless solution receipt. In study 2 , using the chocolate milk receipt vs baseline, we observed an increasing effect after 10 days of treatment with liraglutide; however, this finding was not observed in the analyses using tasteless solution as a comparator. This latter contrast was created to eliminate possible effects of receipt of a solution in general, thus to identify only the added effect of the receipt of palatable solution (i.e. chocolate milk). Nevertheless, we consider the contrast chocolate milk vs baseline also of interest. However, it could be argued that this contrast is less accurate, as it is not corrected for the receipt of a solution in general. However, it is very well possible that, although designed not to do so, the receipt of tasteless solution may also have a certain rewarding effect. This will decrease the quantitative difference in activation within the contrast chocolate milk vs tasteless solution, compared with the more robust contrast using baseline as comparator.

It is to suggest that the central effects of exendin 9-39 and liraglutide may be mediated by their concomitant glucometabolic effects. Both glucose and glucagon have satiating effects in the CNS (Inokuchi et al. 1984, Geary et al. 1992, Page et al. 2011). Although these levels were higher in the T2DM patients and during exendin 9-39 infusion in study 1 , we observed less satiating effects, i.e. reduced central responses to the receipt of chocolate milk. Hence, differences in glucose and glucagon cannot explain our findings in study 1 . In study 2 , differences in glucose levels after 10 days of treatment with liraglutide also cannot explain the effects of liraglutide on the CNS because we found lower glucose levels and enhanced satiating effects of liraglutide. We did not measure glucagon levels in this study. If anything, it could be expected that treatment with liraglutide decreases glucagon levels (Garber et al. 2011), which is associated with lower satiating effects (Inokuchi et al. 1984) and thus expected to reduce the responsiveness to palatable food consumption. Changes in glucagon levels can therefore not explain the observed increased responses after short-term treatment with liraglutide.

It could be argued that the gastro-intestinal side effects of GLP1 and GLP1RA play a role in the central changes in response to palatable food. However, we do not expect gastric emptying to play an important role in our findings. The effects of exendin 9-39 infusion on gastric emptying are subtle and start only 45-60 min. after meal intake (Deane et al. 2010), whereas we started our fMRI measurements $45 \mathrm{~min}$ after meal intake. In addition, during treatment with liraglutide, several patients did report one or more mild gastro-intestinal complaints or nausea; however, these complaints were transient and at the beginning of treatment or after dosage escalation. After 10 days of treatment with liraglutide, i.e. the time point where we observed the effects of liraglutide on the CNS, only two patients reported mild nausea and nausea scores did not differ between treatments. Moreover, the effects of liraglutide on the CNS were similar after exclusion of the two patients with nausea.

In conclusion, healthy lean individuals showed greater CNS activation in response to palatable food consumption, compared with obese T2DM patients. In the healthy lean individuals, we demonstrated that endogenous GLP1 is a physiological signal contributing to central rewarding effects of the consumption of palatable food. Furthermore, treatment with liraglutide improved the reduced CNS activation in obese T2DM patients after short-term treatment, which was associated with body weight loss observed after longer-term treatment. However, long-term effects of GLP1RA treatment on CNS responses to food stimuli were not demonstrated. This may explain why weight loss does not proceed after the initial treatment period with liraglutide $(1.8 \mathrm{mg})$. Taken together, our data emphasize the role for GLP1 in the central processing and regulation of palatable food intake and show a potential mechanism by which treatment with GLP1RA leads to body weight loss.

Supplementary data

This is linked to the online version of the paper at http://dx.doi.org/10.1530/ JOE-15-0461.

\section{Declaration of interest}

M D was a consultant for Abbott, Astra Zeneca, Bristol-Myers Squibb (BMS), Boehringer-Ingelheim, Eli Lilly, GI Dynamics, Inc., Merck Sharp \& Dohme (MSD), Novo Nordisk, Poxel Pharma and Sanofi; speaker for BMS/ Astra Zeneca, Eli Lilly, Novo Nordisk and Sanofi. Through M D, the VU University Medical Center received research grants from Abbott, BMSAstra, Boehringer Ingelheim, Eli Lilly, Medtronic, MSD, Novo Nordisk and Sanofi. R G I J is the principal investigator of studies sponsored by research grants from Novo Nordisk and Eli Lilly. M D and R G I J report receiving no personal payments in connection to the above mentioned activities, but all payments were directly transferred to the Diabetes Center non-profit

Published by Bioscientifica Ltd. 
Research Foundation. J S t K, D J V, L v B, P F C G, E H R and F B declare no conflict of interest.

\section{Funding}

This work was supported in part by a grant from Novo Nordisk. R G I J is financed by the Netherlands Organisation for Scientific Research (NWO) Innovational Research Incentives Scheme Veni (nr. 91613082).

\section{Author contributions}

J S t K designed the study, conducted the experiments, designed the fMRI paradigm, performed data analysis and wrote the manuscript. D J V designed the fMRI paradigm, performed data analysis and wrote the manuscript. $L \vee B$ designed the fMRI paradigm and contributed to writing the manuscript. E H R and P F C G designed the fMRI paradigm and contributed to writing of the manuscript. F B performed the analyses of all structural MRI scans and contributed to writing of the manuscript. M D designed the study. R G I J designed the study, performed data analysis and wrote the manuscript. J S t K and R G I J are the guarantors of this work and, as such, had full access to all the data in the study and take responsibility for the integrity of the data and the accuracy of the data analysis.

\section{Acknowledgements}

The authors thank Sandra Gassman and Ton Schweigmann of the Department of Internal Medicine and Radiology \& Nuclear Medicine of the VU University Medical Center for their assistance during the test visits, as well as the subjects who participated in this study. They thank Nutricia, Zoetermeer, The Netherlands, for providing the Nutridrink.

\section{References}

Blum K, Sheridan PJ, Wood RC, Braverman ER, Chen TJ, Cull JG \& Comings DE 1996 The D2 dopamine receptor gene as a determinant of reward deficiency syndrome. Journal of the Royal Society of Medicine 89 396-400.

Bunck MC, Corner A, Eliasson B, Heine RJ, Shaginian RM, Taskinen MR, Smith U, Yki-Jarvinen H \& Diamant M 2011 Effects of exenatide on measures of beta-cell function after 3 years in metformintreated patients with type 2 diabetes. Diabetes Care 34 2041-2047. (doi:10.2337/dc11-0291)

Buse JB, Rosenstock J, Sesti G, Schmidt WE, Montanya E, Brett JH, Zychma M \& Blonde L 2009 Liraglutide once a day versus exenatide twice a day for type 2 diabetes: a 26-week randomised, parallelgroup, multinational, open-label trial (LEAD-6). Lancet 374 39-47. (doi:10.1016/S0140-6736(09)60659-0)

Chelikani PK, Haver AC \& Reidelberger RD 2005 Intravenous infusion of glucagon-like peptide-1 potently inhibits food intake, sham feeding, and gastric emptying in rats. American Journal of Physiology. Regulatory, Integrative and Comparative Physiology $\mathbf{2 8 8}$ R1695-R1706.

Costantini VJ, Vicentini E, Sabbatini FM, Valerio E, Lepore S, Tessari M, Sartori M, Michielin F, Melotto S, Bifone A, et al. 2011 GSK1614343, a novel ghrelin receptor antagonist, produces an unexpected increase of food intake and body weight in rodents and dogs. Neuroendocrinology 94 158-168. (doi:10.1159/000328968)

Crawley JN \& Beinfeld MC 1983 Rapid development of tolerance to the behavioural actions of cholecystokinin. Nature 302 703-706.
Cummings BP 2013 Leptin therapy in type 2 diabetes. Diabetes, Obesity and Metabolism 15 607-612.

Deane AM, Nguyen NQ, Stevens JE, Fraser RJ, Holloway RH, Besanko LK, Burgstad C, Jones KL, Chapman MJ, Rayner CK, et al. 2010 Endogenous glucagon-like peptide-1 slows gastric emptying in healthy subjects, attenuating postprandial glycemia. Journal of Clinical Endocrinology and Metabolism 95 215-221. (doi:10.1210/jc.2009-1503)

Flint A, Raben A, Astrup A \& Holst JJ 1998 Glucagon-like peptide 1 promotes satiety and suppresses energy intake in humans. Journal of Clinical Investigation 101 515-520.

Frank S, Kullmann S \& Veit R 2013 Food related processes in the insular cortex. Frontiers in Human Neuroscience 7499.

Garber A, Henry RR, Ratner R, Hale P, Chang CT \& Bode B 2011 Liraglutide, a once-daily human glucagon-like peptide 1 analogue, provides sustained improvements in glycaemic control and weight for 2 years as monotherapy compared with glimepiride in patients with type 2 diabetes. Diabetes, Obesity and Metabolism 13 348-356. (doi:10.1111/j.1463-1326.2010.01356.x)

Garner DM \& Olmsted MP 1986 Scoring the eating disorder inventory. American Journal of Psychiatry 143 680-681.

Geary N, Kissileff HR, Pi-Sunyer FX \& Hinton V 1992 Individual, but not simultaneous, glucagon and cholecystokinin infusions inhibit feeding in men. American Journal of Physiology 262 R975-R980.

Hare TA, O’Doherty J, Camerer CF, Schultz W \& Rangel A 2008 Dissociating the role of the orbitofrontal cortex and the striatum in the computation of goal values and prediction errors. Journal of Neuroscience 28 5623-5630. (doi:10.1523/JNEUROSCI.1309-08.2008)

Heppner KM, Kirigiti M, Secher A, Juel PS, Buckingham R, Pyke C, Knudsen LB, Vrang N \& Grove KL 2014 Expression and distribution of glucagon-like peptide-1 receptor mRNA, protein and binding in the male nonhuman primate (Macaca mulatta) brain. Endocrinology 156 255-267. (doi:10.1210/en.2014-1675)

Inokuchi A, Oomura Y \& Nishimura H 1984 Effect of intracerebroventricularly infused glucagon on feeding behavior. Physiology \& Behavior 33 397-400.

Kreymann B, Williams G, Ghatei MA \& Bloom SR 1987 Glucagon-like peptide-1 7-36: a physiological incretin in man. Lancet 2 1300-1304.

Levy LM, Henkin RI, Lin CS, Finley A \& Schellinger D 1999 Taste memory induces brain activation as revealed by functional MRI. Journal of Computer Assisted Tomography 23 499-505.

Merchenthaler I, Lane M \& Shughrue P 1999 Distribution of pre-proglucagon and glucagon-like peptide-1 receptor messenger RNAs in the rat central nervous system. Journal of Comparative Neurology $\mathbf{4 0 3}$ 261-280.

Mojsov S, Weir GC \& Habener JF 1987 Insulinotropin: glucagonlike peptide I (7-37) co-encoded in the glucagon gene is a potent stimulator of insulin release in the perfused rat pancreas. Journal of Clinical Investigation 79 616-619.

O’Doherty J, Rolls ET, Francis S, Bowtell R \& McGlone F 2001 Representation of pleasant and aversive taste in the human brain. Journal of Neurophysiology 85 1315-1321.

Orskov C, Rabenhoj L, Wettergren A, Kofod H \& Holst JJ 1994 Tissue and plasma concentrations of amidated and glycine-extended glucagonlike peptide I in humans. Diabetes 43 535-539. (doi:10.2337/ diab.43.4.535)

Page KA, Seo D, Belfort-DeAguiar R, Lacadie C, Dzuira J, Naik S, Amarnath S, Constable RT, Sherwin RS \& Sinha R 2011 Circulating glucose levels modulate neural control of desire for high-calorie foods in humans. Journal of Clinical Investigation 121 4161-4169. (doi:10.1172/JCI57873)

Pelchat ML, Johnson A, Chan R, Valdez J \& Ragland JD 2004 Images of desire: food-craving activation during fMRI. NeuroImage 23 1486-1493.

Rodriquez de FF, Navarro M, Alvarez E, Roncero I, Chowen JA, Maestre O, Gomez R, Munoz RM, Eng J \& Blazquez E 2000 Peripheral versus central effects of glucagon-like peptide-1 receptor agonists on satiety and body weight loss in Zucker obese rats. Metabolism 49 709-717.

Published by Bioscientifica Ltd. 
Rothemund Y, Preuschhof C, Bohner G, Bauknecht HC, Klingebiel R, Flor H \& Klapp BF 2007 Differential activation of the dorsal striatum by high-calorie visual food stimuli in obese individuals. NeuroImage 37 410-421.

Russell-Jones D, Vaag A, Schmitz O, Sethi BK, Lalic N, Antic S, Zdravkovic M, Ravn GM \& Simo R 2009 Liraglutide vs insulin glargine and placebo in combination with metformin and sulfonylurea therapy in type 2 diabetes mellitus (LEAD-5 met+SU): a randomised controlled trial. Diabetologia 52 2046-2055. (doi:10.1007/ s00125-009-1472-y)

Schick RR, Zimmermann JP, vorm Walde T \& Schusdziarra V 2003 Peptides that regulate food intake: glucagon-like peptide 1-(7-36) amide acts at lateral and medial hypothalamic sites to suppress feeding in rats. American Journal of Physiology. Regulatory, Integrative and Comparative Physiology 284 R1427-R1435.

Schroevers MJ, Sanderman R, van SE \& Ranchor AV 2000 The evaluation of the Center for Epidemiologic Studies Depression (CES-D) scale: depressed and positive affect in cancer patients and healthy reference subjects. Quality of Life Research 9 1015-1029.

Schwartz MW, Woods SC, Porte D Jr, Seeley RJ \& Baskin DG 2000 Central nervous system control of food intake. Nature $\mathbf{4 0 4} 661-671$.

Secher A, Jelsing J, Baquero AF, Hecksher-Sorensen J, Cowley MA, Dalboge LS, Hansen G, Grove KL, Pyke C, Raun K, et al. 2014 The arcuate nucleus mediates GLP1 receptor agonist liraglutidedependent weight loss. Journal of Clinical Investigation $\mathbf{1 2 4}$ 4473-4488. (doi:10.1172/JCI75276)

Shin YK, Martin B, Golden E, Dotson CD, Maudsley S, Kim W, Jang HJ Mattson MP, Drucker DJ, Egan JM, et al. 2008 Modulation of taste sensitivity by GLP1 signaling. Journal of Neurochemistry $\mathbf{1 0 6}$ 455-463.

Shugrue PJ, Lane M \& Merchenthaler I 1996 Glucagon-like peptide-1 receptor (GLP1-R) mRNA in the rat hypothalamus. Endocrinology 137 5159-5162.

Sisley S, Gutierrez-Aguilar R, Scott M, D'Alessio DA, Sandoval DA \& Seeley RJ 2014 Neuronal GLP1R mediates liraglutide's anorectic but not glucose-lowering effect. Journal of Clinical Investigation $\mathbf{1 2 4}$ 2456-2463. (doi:10.1172/JCI72434)

Sloan JH, Siegel RW, Ivanova-Cox YT, Watson DE, Deeg MA \& Konrad RJ 2012 A novel high-sensitivity electrochemiluminescence (ECL) sandwich immunoassay for the specific quantitative measurement of plasma glucagon. Clinical Biochemistry 45 1640-1644. (doi:10.1016/j. clinbiochem.2012.07.111)

Small DM, Zatorre RJ, Dagher A, Evans AC \& Jones-Gotman M 2001 Changes in brain activity related to eating chocolate: from pleasure to aversion. Brain 124 1720-1733.

Stice E, Spoor S, Bohon C \& Small DM $2008 a$ Relation between obesity and blunted striatal response to food is moderated by TaqIA A1 allele. Science 322 449-452. (doi:10.1126/science.1161550)

Stice E, Spoor S, Bohon C, Veldhuizen MG \& Small DM $2008 b$ Relation of reward from food intake and anticipated food intake to obesity: a functional magnetic resonance imaging study. Journal of Abnormal Psychology 117 924-935. (doi:10.1037/a0013600)

Stoeckel LE, Weller RE, Cook EW III, Twieg DB, Knowlton RC \& Cox JE 2008 Widespread reward-system activation in obese women in response to pictures of high-calorie foods. NeuroImage 41 636-647. (doi:10.1016/j.neuroimage.2008.02.031)
Szayna M, Doyle ME, Betkey JA, Holloway HW, Spencer RG, Greig NH \& Egan JM 2000 Exendin-4 decelerates food intake, weight gain, and fat deposition in Zucker rats. Endocrinology 141 1936-1941.

Tang DW, Fellows LK, Small DM \& Dagher A 2012 Food and drug cues activate similar brain regions: a meta-analysis of functional MRI studies. Physiology \& Behavior 106 317-324. (doi:10.1016/j. physbeh.2012.03.009)

Ten Kulve JS, Veltman DJ, van Blomendaal L, Barkhof F, Deacon CF, Holst JJ, Konrad RJ, Sloan JH, Drent ML, Diamant M, et al. 2015a Endogenous GLP1 mediates postprandial reductions in activation in central reward and satiety areas in patients with type 2 diabetes. Diabetologia 58 2688-2698. (doi:10.1007/s00125-015-3754-x)

Ten Kulve JS, Veltman DJ, van Blomendaal L, Barkhof F, Drent ML, Diamant M \& IJzerman RG 2015b Liraglutide reduces CNS activation in response to visual food cues only after short-term treatment in patients with type 2 diabetes. Diabetes Care 39 214-221. (doi:10.2337/ dc15-0772)

Trinko R, Sears RM, Guarnieri DJ \& DiLeone RJ 2007 Neural mechanisms underlying obesity and drug addiction. Physiology \& Behavior $\mathbf{9 1}$ 499-505.

Turton MD, O'Shea D, Gunn I, Beak SA, Edwards CM, Meeran K, Choi SJ, Taylor GM, Heath MM, Lambert PD, et al. 1996 A role for glucagonlike peptide-1 in the central regulation of feeding. Nature 379 69-72.

van Bloemendaal L, IJzerman RG, Ten Kulve JS, Barkhof F, Konrad RJ, Drent ML, Veltman DJ \& Diamant M 2014 GLP1 receptor activation modulates appetite- and reward-related brain areas in humans. Diabetes 63 4186-4196. (doi:10.2337/db14-0849)

van Bloemendaal L, Veltman DJ, Ten Kulve JS, Groot PF, Ruhe HG, Barkhof F, Sloan JH, Diamant M \& IJzerman RG 2015 Brain rewardsystem activation in response to anticipation and consumption of palatable food is altered by GLP1 receptor activation in humans. Diabetes, Obesity and Metabolism 17 878-886. (doi:10.1111/ dom.12506)

Verdich C, Flint A, Gutzwiller JP, Naslund E, Beglinger C, Hellstrom PM, Long SJ, Morgan LM, Holst JJ \& Astrup A 2001 A meta-analysis of the effect of glucagon-like peptide-1 (7-36) amide on ad libitum energy intake in humans. Journal of Clinical Endocrinology and Metabolism $\mathbf{8 6}$ 4382-4389.

Vilsboll T, Christensen M, Junker AE, Knop FK \& Gluud LL 2012 Effects of glucagon-like peptide-1 receptor agonists on weight loss: systematic review and meta-analyses of randomised controlled trials. BMJ $\mathbf{3 4 4}$ d7771. (doi:10.1136/bmj.d7771)

Volkow ND \& Wise RA 2005 How can drug addiction help us understand obesity? Nature Neuroscience 8 555-560.

Wang GJ, Volkow ND, Telang F, Jayne M, Ma J, Rao M, Zhu W, Wong CT, Pappas NR, Geliebter A, et al. 2004 Exposure to appetitive food stimuli markedly activates the human brain. NeuroImage $\mathbf{2 1}$ 1790-1797. (doi:10.1016/j.neuroimage.2003.11.026)

Wang GJ, Volkow ND, Thanos PK \& Fowler JS 2009 Imaging of brain dopamine pathways: implications for understanding obesity. Journal of Addiction Medicine 3 8-18.

Williams DL, Baskin DG \& Schwartz MW 2009 Evidence that intestinal glucagon-like peptide-1 plays a physiological role in satiety. Endocrinology 150 1680-1687.

Zald DH \& Pardo JV 2000 Cortical activation induced by intraoral stimulation with water in humans. Chemical Senses 25 267-275.

Received in final form 28 December 2015

Accepted 14 January 2016 http://joe.endocrinology-journals.org

DOI: 10.1530/JOE-15-0461
C 2016 Society for Endocrinology Printed in Great Britain 